\title{
Characterization of hormonal changes in patients with dystrophic diseases of the vulva
}

\begin{abstract}
The article contains a review of the literature and its own data on the dyshormonal aspect of the pathogenesis of scleroatrophic lichen and dystrophic diseases of the vulva (DDV) as a whole. The authors describe the age-related mechanisms of gradual reduction of the main sex hormones, as well as their influence on the skin structure of the female genitalia. Based on the results of our own research, conclusions were drawn about the unreliable difference in the decrease in the estradiol content in perimenopausal patients with DDV and without DDV. The authors also recorded a prevailing hypothyroidism pattern in patients with DDV, and a similar pattern in patients of perimenopausal age without DDV, which may be due to regional lack of iodine in the environment. The authors suggested that the background role of an imbalance of thyroid hormones in the genesis of the DDV. The necessity of an individual approach to determining the level of estradiol and thyroid hormones in patients with dystrophic diseases of the vulva is substantiated.
\end{abstract}

Keywords: DDV, hormonal changes, natural moisturizing factor, hormone replacemen therapy
Volume 7 Issue 5 - 2019

\author{
Porsokhonova DF, Rakhmatullaeva SN, \\ Republican Specialized Research Medical Center of \\ Dermatovenerology and Cosmetology, Ministry of Health of the \\ Republic of Uzbekistan, Uzbekistan \\ Correspondence: Porsokhonova DF, Republican Specialized \\ Research Medical Center of Dermatovenerology and \\ Cosmetology, Ministry of Health of the Republic of Uzbekistan, \\ Uzbekistan,Email delya.porsokhonova@mail.ru
}

Received: August 23, 2019 | Published: September 06, 2019

\section{Introduction}

The problems of the postmenopausal period have acquired special significance in recent years in connection with a significant increase in the life expectancy of women, which is especially clearly seen in developed countries. ${ }^{1}$ However, along with this, that period of life, due to which its duration increases, is accompanied by age-related involutive changes, the development of a number of pathological symptoms, syndromes and diseases that worsen the quality of life. ${ }^{2}$ One of these diseases, most often encountered at the peri- and postmenopausal age, is sclerosis of the vulva (syn.: scleroatrophic lichen, kraurosis of the vulva, etc.), a pathological process characterized by progressive sclerosis and atrophy of the external genital organs, accompanied by symptoms of neuroendocrine and psychoemotional disorders. ${ }^{3}$ Age-related, genetically determined changes in hormonal homeostasis and the reproductive function of women can also cause a number of changes in the genital area, which are based on physiological disorders in neuroendocrine regulation.

One of the leading factors in the origin of postmenopausal sclerotic lichen is the theory of estrogen deficiency. ${ }^{4}$ In the etiology of sclerotic lichen, an important role is also given to changes at the level of tissue hormone receptors, the deficiency of which plays an important role in the development of dystrophic diseases of the vulva (DDV). The hormonal status of postmenopausal women is characterized by early estrogen deficiency, an excess of testosterone, at the initial stage FSH with its subsequent acute deficiency, imbalance between hormones of other endocrine systems - adrenal glands, thyroid gland and pituitary gland. In the process of age-related biological transformation, a decrease in the functional activity of the hypothalamic-pituitarygonadal system, steroidogenesis deficiency in general, and a decrease in the secretion of estrogen/progesterone pool lead to pronounced changes in connective tissue structures. Destructive processes in the derma are a pathognomonic sign of late menopause. A decrease in the percentage of estrogens in the skin during menopause leads to a decrease in the rate of keratinopoiesis, a decrease in the proliferative activity of keratinocytes and a disruption in the metabolism of epidermal lipids. The distribution density of fibroblasts, in which degradation of the synthesis of procollagen and tissue inhibitors of metalloproteinase is also observed, decreases. A decrease in the content of elastin and proteoglycans, as well as a gradual disintegration of elastin fibers, leads to sagging and wrinkling skin. A decrease in the percentage of dermatinsulfate and hyaluronic acid is the etiological cause of a decrease in the concentration of natural moisturizing factor (NMF) in the range of $20-30 \%$ for every 10 years of life. Hormonal insufficiency and a decrease in the excretion of sex steroids have a direct effect on the functional activity of cytoplasmic estrogen receptors of the skin, and sclerosis of microcapillaries and atrophy of the follicular apparatus against the background of impaired moisture exchange and architectonics of the collagen-elastin skeleton of the derma form a typical clinical picture of senile revolution and postmenopausal women - dryness and thinning of the skin, wrinkling, age-related hyper- and depigmentation. Literature indicates a wide variability of the level of receptors in the vulva tissue, in particular with sclerotic lichen..$^{5-9}$ however, these messages, in addition to the inconsistency of data, contain very few clinical observations. At the same time, knowledge of the receptor status of vulvar tissue in this pathology in postmenopausal patients allows for a more differentiated construction of hormone replacement therapy for this disease. For the adequate realization of the hormonal effect, it is necessary not only the presence of hormones in the peripheral blood, but also a sufficient level of specific receptors in target tissues. ${ }^{10,11}$ The response of tissues to the effects of steroid hormones is determined by the concentration of receptors, their structure and properties of hormones. The local effect of steroid hormones is realized by passive diffusion of them into the cells of the body. Delayed only in cells of sensitive tissues, they form complexes with cytosolic receptors, followed by translocation into the cell nucleus.

In connection with the foregoing, theoretically, estriol-containing drugs should play a priority role in the choice of therapy for atrophic disorders caused by a decrease in gonadal function. ${ }^{4,12}$ This is due to 
the specific local sensitivity of the tissues of the lower parts of the urogenital tract to estriol. It is generally accepted that the appointment of hormone replacement therapy (HRT), which is based on modern ideas about the ethiopathogenetic concept of the menopause, is considered an important condition for the effectiveness of conservative measures in the treatment of dermatological pathology during menopause. According to published data, in the USA and Germany, a quarter of women aged 45-64 use HRT, in Finland and Australia - 30\% and 28\%, in Brazil and Italy - 5\% respectively. Global medical statistics record an upward trend in HRT intake in almost all regions of the planet.

However, hormone therapy for sclerotic lichen is far from effective in all cases. There is also evidence of the adverse effects of even local estrogens on breast and endometrial tissue. In this regard, the appointment of estriol-containing drugs requires a thorough preliminary analysis of the possible causes of the occurrence of ADD, taking into account contraindications and side effects of steroid therapy.

The use of traditional means of conservative therapy in the treatment of these diseases of the genital skin in most does not bring the expected clinical result. In recent years, the use of mesotherapy has been actively begun both for the purpose of correcting the aesthetics of the vulva and for the treatment of various manifestations of ADV. However, the methods of correction of involutionally altered skin used in modern dermatology are based on eliminating only visible agerelated changes, while specialists at the practical level do not always take into account the degree of hormonal dysfunction, menopause and other factors in the development of senile degeneration of the genital skin. The role of infectious agents in connection with the scarcity of inflammatory manifestations associated with age-related involution of stratified vaginal epithelium and a decrease in the level of lactobacilli is practically not taken into account. The low adherence of patients with ADD to treatment, associated with the intimate nature of the problem, as well as the localization of pruritus in the external genital area, constricts the diagnostic search and does not contribute to a full microbiological endocrinological and pathomorphological examination, which should be carried out in each case. In this regard, the aim of our study was to determine the content of thyroid hormones and some sex hormones in patients with dystrophic diseases of the vulva.

\section{Material and methods}

Under observation were 40 women aged from 45 to 60years (average age was $52.1 \pm 0.01$ years). The comparison group consisted of 20 practically healthy women of a similar age without vulvar pathology. The hormone content was also compared with the age standards of healthy women of reproductive age. All women underwent a study of hormonal status. In the blood serum, the content of estradiol (E2), progesterone, as well as thyroid stimulating hormone (TSH), thyroxine (T4) and triiodothyronine (T3) was determined by enzyme-linked immunosorbent assay (ELISA).

\section{Results}

With increasing age, the level of estradiol (E2) progressively decreased in comparison with the control group, both in the patients of the main and comparison groups. There were no statistically significant differences in the estradiol content between the main and comparison groups. The progesterone content did not undergo such sharp fluctuations.

\begin{tabular}{|c|c|c|c|}
\hline $\begin{array}{l}\text { Test } \\
\text { hormones }\end{array}$ & $\begin{array}{l}\text { Main group (women } \\
\text { with ADV) } \\
n=40\end{array}$ & $\begin{array}{l}\text { Comparison group (almost healthy } \\
\text { women of a similar age) } \\
n=38\end{array}$ & $\begin{array}{l}\text { Control group (healthy women of } \\
\text { reproductive age) } \\
n=35\end{array}$ \\
\hline Estradiol & $7 I, I \pm 2,59 \mathrm{nmol} / \mathrm{L}$ & $97,0 \pm 0,80 \mathrm{nmol} / \mathrm{L}^{\circ \circ \circ}$ & $324,0 \pm 18,67 \mathrm{nmol} / L^{*}$ \\
\hline Progesterone & $0.6 \pm 0.01 \mathrm{nmol} / \mathrm{L}$ & $0.7 \pm 0.01 \mathrm{nmol} / \mathrm{L}^{\circ \circ \circ \circ}$ & $34.0 \pm 3.34 \mathrm{nmol} / \mathrm{L}^{*}$ \\
\hline T3 & $1.2 \pm 0.03 \mathrm{nmol} / \mathrm{L}$ & $\mathrm{I}, 9 \pm 0,0 \mathrm{Inmol} / \mathrm{L}^{\circ \circ \circ \circ}$ & $\mathrm{I}, 6 \pm 0,0 \mathrm{Inmol} / \mathrm{L} * * * *$ \\
\hline T4 & $80,5 \pm 1,53 \mathrm{nmol} / \mathrm{L}$ & $\mathrm{I} / 0,98 \pm 0,79 \mathrm{nmol} / \mathrm{L}^{\circ \circ \circ}$ & $106,0 \pm 0,84 \mathrm{nmol} / \mathrm{L}^{* * *}$ \\
\hline TTG & $5.2 \pm 0.38 \mathrm{mlU} / \mathrm{ml}$ & $3,3 \pm 0,12 \mathrm{mlU} / \mathrm{ml}^{\circ}$ & $\mathrm{I}, 3 \pm 0,02 \mathrm{mlU} / \mathrm{ml} * * * * *$ \\
\hline
\end{tabular}

Note:*-differences between the main and control groups are statistically significant $(\mathrm{P} \leq 0.05)$;

$*_{p}<0.05 ; * *_{-}-p<0.01 ; * * *-p<0.001$; $* * * *$ - $p>0.05$;

${ }^{\circ}$-the differences between the main and comparison groups are statistically significant $(\mathrm{P} \leq 0.05)$;

${ }^{\circ} \mathrm{P}<0.05 ;{ }^{\circ 0}-\mathrm{P}<0.01 ;{ }^{\circ 00}-\mathrm{P}<0.001^{\circ 000}-\mathrm{P}>0.05$.

The content of thyroid hormones (T3, T4) in women of the main group was below normal. A statistically significant decrease in T3 was detected in patients of the main group, both in relation to the comparison group and in relation to the control group. T4 level underwent a similar dynamics, having a minimum value also in patients of the main group. The level of TSH was quite high in both study groups (the main and the comparison group versus the control group). The above parameters indicate a decrease in thyroid function both in the comparison group and in the main group, however, in the main group they were statistically significant.

\section{Findings}

Progressive estradiol E2 deficiency, beginning long before the last menstruation in a woman's life, reaching its peak in the postmenopausal period, occurred in patients with ADD with approximately the same frequency as in women without ADD, which indicates the questionable role of estrogen deficiency in the origin of ADD. In the women examined by us, the concentration of progesterone did not undergo significant changes. A high percentage of hypothyroidism in perimenopausal women with ADD in the region may be due to iodine deficiency in the environment. The endemic nature of thyroid damage in the republic contributes to earlier menstrual dysfunction and the onset of menopause earlier, compared with women without thyroid pathology, which can affect the condition of the genital skin. However, a similar situation with a reduced state of thyroid hormones in patients of perimenopausal age without ADD is more likely to indicate the background role of an imbalance of thyroid hormones in the genesis of ADD. 


\section{Acknowledgments}

None.

\section{Conflicts of interest}

The authors declare that there are no conflicts of interest.

\section{Funding}

None.

\section{References}

1. Malyshev LN. Neuroendocrine aspects of vulvar kraurosis. Kazan, 1981 Abstracts of reports of a republican scientific and practical conference. 1981;69-70.

2. Rozhinskaya L Ya, Ermakova IP, Marova EI, et al. Phosphoric calcium metabolism and calcium regulating hormones in hypercorticism. Problems of endocrinology. 1986;32(6):13-18.

3. Ayahan A, Tuncer ZC, Kaya H. Vulvar dystrophy: en evaluation of 285 cases. Eur J Gynecol.Oncol. 1997;18(2):139-140.

4. Ashrafyan LA, Kharchenko NV, Kiselev VI, et al. Cancer of the vulva: epiopathogenetic concept. M. 2006. 192. p.
5. Kiryu H, Ackerman AB. A critique of current classifications of vulvar diseases. Am J Dermatopathol. 1990;12(4):377-392.

6. Kuhl H. Pharmacokinetics of oestrogens and progestagens. Maturitas. 1990;12(3):171-197.

7. Pinzger G, Heim K. Diagnosis and therapy of vulvar dystrophy. Gynacol Rundsch. 1991;31(S2):225-229.

8. Sodo G, Pizzo A. Vulvar dystrophy. Minerva Gynecol. 1990;42(6):251-262

9. Tsai MJ, Clark J, Schrader WT, et al. Mechanisms of action of hormones that act as transcription-regulatory factors. In: JD Wilson, editor. Williams textbook of endocrinology. Philadelphia, WB Saunders Coтр. 1998;55-87.

10. Sidorenko S Yu. Endocrine factors in the development of leukoplakia and vulvar kraurosis. Questions of oncology. 1990;36(10):1210-1214.

11. Estimator VP. Menopausal disorders and the principles of hormone replacement therapy. Therapist, archive. 1995;10:70-74.

12. Burpgein LM. Estrogens, aging and age-related pathology. Successes in gerontology. 1998;2:90-97. 